\title{
An Expert Selection System for Medical Diagnosis
}

\author{
Shaly K K ${ }^{1}$, Shahana $\mathbf{T}^{2}$ \\ ${ }^{1}$ MTech CSE, AWH Engineering College, Calicut, India \\ ${ }^{2}$ Assistant Professor, AWH Engineering College, Calicut, India
}

\begin{abstract}
This paper introduces an expert selection system that uses learning and finds the best expert to assign to each patient based on the context of the patient. The context may include a variety of information related to the patient's health condition, age, gender, previous drug doses etc. In this the most relevant information is embedded in only a few contexts. The relevant contexts may be different for different health conditions.A new class of algorithms were introduced for discovering the most relevant contexts and the best clinic and expert to use to make a diagnosis given a patient's contexts. The problem with the existing system is that it uses Learn the EXpert algorithm (LEX) which assigns a single expert to each patient, whether an own expert of the clinic or another clinic's expert. The proposed system assigns multiple experts to patients with complex diseases.
\end{abstract}

Keywords: Context Adaptive algorithm, clinical decision support systems, healthcare informatics

\section{Introduction}

This paper includes an expert selection system that learns online the best expert to assign to each patient depending on the context of the patient. In general, the context can include an enormous number and variety of information related to the patient's health condition, age, gender, previous drug doses, and so forth, but the most relevant information is embedded in only a few contexts. If these most relevant contexts were known in advance, learning would be relatively simple but they are not. Moreover, the relevant contexts may be different for different health conditions. To address these challenges, a new class of algorithms were introduced for discovering the most relevant contexts and the best clinic and expert to use to make a diagnosis given a patient's contexts.

As the number of patients grows, the context adaptive algorithm will discover the optimal expert to select for patients with a specific context. The problem with the existing system is that ,it uses Learn the EXpert algorithm (LEX) which assigns a single expert to each patient, whether an own expert of the clinic or another clinic's expert. In current clinical practice, patients are referred to experts in an ad-hoc manner based on one or more of the following factors: signs and symptoms of the patient, patient's or primary care physician's preference, insurance plan, and availability of the physician.

This paper develops a framework and associated methods and algorithms that uses semantic knowledge about the patient to assess and recommend expertise with the goal of optimizing the process for diagnosing a patient .Assume that the diagnostic accuracy of an expert (either human or CDSS) depends on the context of the patient for which the decision is made. This context is all the information pertaining to the patient under consideration that can be utilized in the decision making process.

Sometimes, rural clinic may have only a primary care physician (PCP), a registered nurse and equipment, but no specialist or CDSSs and may be able to request information from a more established hospital which implements multiple
CDSSs and has several experienced human experts. Hence, based on the context of the patient, each clinic learns whether it should rely on its own experts or request another clinic to make diagnostic decisions.

The methodology in this paper learns the most relevant context(s) pertinent to the current health condition of the patient and uses it/them to estimate the level of expertise exhibited by the expert. The level of expertise is defined based on the accuracy of their diagnostic. Moreover, different clinics have healthcare professionals with different expertise and some of these clinics may have access to CDSSs from different manufacturers and of different types while some others just rely on human experts. In the proposed system, these clinics can cooperate with each other to improve diagnostic accuracy by learning the contextual specializations of the other clinics.

\section{Literature Survey}

There are a number of works related on Expert Selection systems. It is demonstrated in[1]that the general philosophy for the use of ANN(Artificial Neural Networks) in diagnostic approaches through selected examples, documenting the enormous variability of data that can serve as inputs for ANNs. Each type of data provides information that must be evaluated and assigned to a particular pathology during the diagnostic process.

An ANN is a mathematical representation of the human neural architecture, reflecting its learning and generalization abilities. For this reason, ANNs belong to the field of artificial intelligence. ANNs are widely applied in research because they can model highly non-linear systems in which the relationship among the variables is unknown or very complex. A neural network is formed by a series of neurons (or nodes) that are organized in layers. Each neuron in a layer is connected with each neuron in the next layer through a weighted connection. The value of the weight Wij indicates the strength of the connection between the ith neuron in a layer and the jth neuron in the next one. 


\section{International Journal of Science and Research (IJSR) \\ ISSN (Online): 2319-7064}

Index Copernicus Value (2013): 6.14 | Impact Factor (2015): 6.391

The neurons in the input layer receive the data and transfer them to neurons in the first hidden layer through the weighted links. Here, the data are mathematically processed and the result is transferred to the neurons in the next layer. Ultimately, the neurons in the last layer provide the networks output.

It is proposed in [2] that decision support systems that have been developed to assist physicians in the diagnostic process. often are based on static data which may be out of date. A decision support system which can learn the relationships between patient history, diseases in the population, symptoms, pathology of a disease, family history and test results, would be useful to physicians and hospitals.

Decision support systems are defined as interactive computer based systems intended to help decision makers utilize data and models in order to identify problems, solve problems and make decisions. They incorporate both data and models and they are designed to assist decision makers in semi-structured and unstructured decision making processes. They provide support for decision making, they do not replace it. The mission of decision support systems is to improve effectiveness, rather than the efficiency of decisions.

It is demonstrated in [3] that a framework has been proposed for the implementation of a hybrid system which uses both RBR and CBR for medical diagnosis. The traditional method of reasoning was rule-based reasoning (RBR). It does not use past experiences to reason. Case based reasoning (CBR), on the other hand uses past experiences to derive results for new cases.

It is proposed in [4] that a system called DExS is for dealing with the problem of disease diagnosis is an expert system. Computer based methods are increasingly used to improve the quality of medical services. Artificial Intelligence (AI) is the area of computer science focusing on creating expert machines that can engage on behaviors that humans consider intelligent. An expert system is a system that employs human knowledge captured in a computer to solve problems that ordinarily require human expertise.

Expert system seeks and utilizes relevant information from their human users and from available knowledge bases in order to make recommendations. The data and knowledge of DExS are collected from different sources. The first primary source is the medical knowledge of expert doctors. The second source is from specialized databases, books and a few electronic websites.

\section{Problem definition}

In the current setting the clinics cooperate with each other by making diagnosis recommendations for each other's patients when requested. Such cooperation is very beneficial when the expertise of the experts vary among the clinics .However, the problem with the existing system is that, LEX assigns a single expert to each patient (whether an own expert of the clinic or another clinic's expert).In some clinical applications, such as some complex diseases, multiple experts and clinics can work simultaneously to diagnose a patient, which can significantly improve the diagnosis accuracy.

\section{Methodology}

This section includes the system architecture and working principle of the proposed system.

\subsection{System Architecture}

The proposed system includes mainly three modules. The first module is the Support System which includes the Information Processing Engine and a Primary Care Physician(PCP). When a patient with a given context(context may be age, sex, other health conditions etc.) arrives ,then the Support System finds the relevant contexts, that is the contexts that are necessary to Diagnose the Patient. Also there is a Primary Care Physician who initially examines the patient.

The second module is the Expert Selection module. The relevant contexts are analysed by the Expert selection module, the expert may be a Human Expert or a Clinical Decision Support System (CDSS).The Expert selection module assigns the best expert based on the context. If multiple experts are necessary to diagnose certain complex diseases, then the system assigns multiple experts for a single patient.

The Expert Selection module includes a number of clinics. The experts selected may be from a single clinic or from more than one clinics. If an expert for a particular patient is not available in a clinic $i$ then the system can forward the patient to other clinics, say $\mathrm{j}, \mathrm{k}$ etc.

The diagnosis action is analysed by the Primary Care Physician and then gives the diagnosis recommendations to the patient. The third module is the learning module. Based on the context of the patient, each clinic learns whether it should rely on its own experts or request another clinic to make diagnostic decisions. If there is some other clinic $j$ works based on the contexts of the patient approaching the system, then the system learns from clinic j. Clinics can cooperate with each other to improve diagnostic accuracy by learning the contextual recommendations of the other clinics.

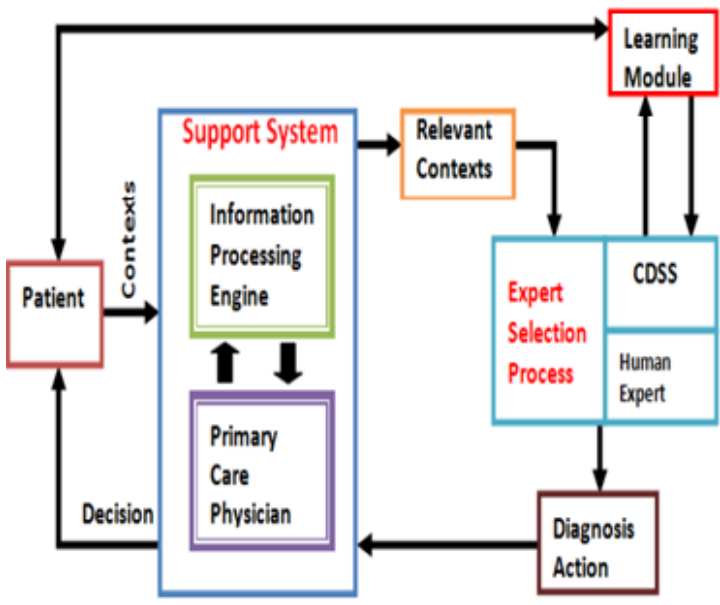

Figure 1: System Architecture 


\section{International Journal of Science and Research (IJSR)}

ISSN (Online): 2319-7064

Index Copernicus Value (2013): 6.14 | Impact Factor (2015): 6.391

\subsection{Working Principle}

This section describes the basic steps used in this paper to implement the system.

To Find the Experts.

INPUT:A database $\mathrm{D}=\mathrm{d} 1, \mathrm{~d} 2 \ldots \mathrm{dn}$ containing $\mathrm{n}$ contexts and a disease symptom set.

OUTPUT: A set of K clusters.

1. Identify disease by matching clusters of symptoms with disease symptom tree.

2. Ranking of clusters based on weight.

3. Output possible health issues

4. Apply the Expert selection algorithm

5. If there is no human expert to diagnose a particular disease then CDSS(Clinical Decision Support System) chooses the best decision(suggestion) .

6. Send the suggestions to Primary Care Physician(PCP).

Expert Selection Algorithm

INPUT: Set of experts and practice records, health issues or relevant contexts

1. Select doctors based on health issues and related department in a particular clinic,say clinic i

2. Forward contexts to chosen doctors based on expert ranking system.

3. If multiple experts are necessary to diagnose a disease then assign multiple experts for a single patient

4. Verify suggestions from experts

5. Rerank experts based on false and correct decisions.

6. Update health records

\section{Results and Discussions}

The system learns online the best clinic and expert to assign to each patient depending on the context. The existing system uses a new class of algorithms called learn the EXpert algorithm aimed at discovering the most relevant contexts and the best clinic and expert to use to make a diagnosis given a patient's contexts.

The problem with the existing system is that ,it uses Learn the EXpert algorithm (LEX)which assigns a single expert to each patient (whether an own expert of the clinic or another clinic's expert).In some clinical applications, such as some complex diseases, multiple experts and clinics can work simultaneously to diagnose a patient, which can significantly improve the diagnosis accuracy.

The system assigns multiple experts to patients with complex diseases. As the number of patients grows and as the number of experts registered increases the efficiency of the system increases.The proposed context adaptive algorithm will discover the optimal expert to select for patients with a specific context. Since multiple experts are selected compared to the existing system the diagnostic accuracy of the proposed system is high. As the number of patients increases the scalability of the proposed system will also high.

\section{Conclusion}

The existing system is a context adaptive medical diagnosis system that selects from a pool of human experts and CDSSs to make diagnosis recommendations. The system learns online, which context of the patient to use, and which expert to rely on when making diagnosis recommendations. The system uses the algorithm LEX which learns the best expert for treating a patient with a specific context not only within a clinic but also across all clinics; hence, its performance is better than the performance of the best expert within any given clinic. The system assigns multiple experts to patients with complex diseases. Various related papers of the proposed system were studied and the system architecture of the proposed system Expert Selection System for Medical Diagnosis is framed.

\section{Future Work}

Future work proposed is, designing algorithms that can track the changes in a clinician's performance by taking into account of patient's history, feedback etc. Also, to incorporate more evaluation methods on expert's performance of the system.

\section{References}

[1] Filippo Amato1,Alberto Lopez,Josef Havel1,"Artificial neural networks in medical diagnosis", Journal of applied biomedicine pp.47-58 2013.

[2] D.Senthil Kumar, G.Sathyadevi and S.Sivanesh "Decision Support System for Medical Diagnosis Using Data Mining".International Journal of Computer Science Issues, Vol. 8, Issue 3, No. 1, May 2011

[3] Deepti Anne John, Rose Rani John.,"A Framework for Medical Diagnosis using Hybrid Reasoning". Proceedings of the international multiconference of engineers and computer scientists 2010 vol.1.

[4] P.Santosh Kumar Patra,Dipti Prava Sahu,Indrajit Mandal,"An Expert System for Diagnosis of Human Diseases".International Journal of Computer Applications (0975 8887) Volume 1 No. 13

[5] Shweta Kharya," Using Data mining techniques for Diagnosis and Prognosis of Cancer disease" International Journal of Computer Science, Engineering and Information Technology,Vol.2,No.2,April 2012

\section{Author Profile}

Shaly K K received the B.Tech degree in Computer Science \& Engineering from Cochin University of Science \& Technology (CUSAT) in 2005. During 2005-2013, she worked as lecturer in various engineering colleges. She now doing final semester, M.Tech in Computer Science \& Engineering from Calicut University.

Shahana T received the B.Tech degree in Computer Science \& Engineering from Calicut University in 2004. Also she completed her M.Tech in Computer Science \& Engineering from Calicut University in 2012. She is working as Asst. Professor in Dept.of Computer Science \& Engineering at AWH Engineering College Kozhikode for the past 10 years. 